\title{
Parâmetros demográficos de Micoureus demerarae (Didelphidae, Marsupialia) em áreas contíguas de manguezal e terra Firme, Bragança, Pará, Brasil
}

\author{
Fernanda A. G. Andrade; Marcus E. B. Fernandes \& Stélio A. C. Brito \\ Laboratório de Ecologia de Manguezal, Universidade Federal do Pará. Campus de Bragança, 68600-000 Bragança, Pará, \\ Brasil. E-mail: mebf@ufpa.br
}

\begin{abstract}
Demographic parameters of Micoureus demerarae (Didelphidae, Marsupialia) in contiguous mangrove and terra firme areas, in Bragança, Pará, Brazil. This study investigated some aspects of the population dynamics of Micoureus demerarae (Thomas, 1905), such as: density, recruitment, and survivorship in two contiguous ecosystems, mangal and terra firme at the Fazenda das Salinas in the northeast of the state of Pará. One transect in each system was used for fieldwork. Data collection was carried out from November 2002 to September 2003. The following population parameters were estimated: density, recruitment, and survivorship. The results defined an open population with an even distribution for individuals from mangal and a clumped one for individuals from terra firme, and the evident seasonality with higher proportion of males in the terra firme. The reproductive period was the most important parameter to the population fluctuation in the beginning of the dry season. The environmental impacts over the population dynamics parameters of $M$. demerarae seem to be determinant for the maintenance of these populations.

KEY WORDS. Demography; long-furred woolly mouse opossum; mangrove forest; Brazilian Amazon.
\end{abstract}

\begin{abstract}
RESUMO. Este estudo investigou alguns variáveis populacionais da espécie Micoureus demerarae (Thomas, 1905), que incluem: densidade, recrutamento e sobrevivência. Sendo que, os espécimes coletados foram obtidos em dois ecossistemas contíguos, manguezal e terra firme da denominada Fazenda das Salinas, a qual esta localizada próxima ao município de Bragança, no nordeste do Estado do Pará. Para tanto, foram abertas duas transecções em cada ambiente destinadas as coletas realizadas de novembro de 2002 a setembro de 2003. Os resultados definiram uma população aberta com distribuição uniforme para os indivíduos do manguezal e agregada para os da terra firme, além de evidente sazonalidade, com maior proporção de machos na terra firme. O período reprodutivo foi o parâmetro mais importante para a flutuação populacional no início da estação seca. Os impactos ambientais sobre os parâmetros da dinâmica populacional de $M$. demerarae parecem ser determinantes na manutenção dessas populações da Fazenda das Salinas.
\end{abstract}

PALAVRAS-CHAVE. Demografia; cuíca-branca; floresta de mangue; Amazônia brasileira.

Micoureus demerarae (Thomas, 1905) é a espécie de maior porte desse gênero, já tendo sido considerada sinônimo ou subgênero de Marmosa (Gray, 1821), chegando a ser identificada como Marmosa cinerea (GARDNER 1993, NowACK 1999). Das características peculiares de $M$. demerarae destacam-se o hábito arborícola e a variabilidade de itens na sua dieta, chegando a ser classificada como uma espécie insetívora-onívora (Leite et al. 1994, NowAK 1999). Outro aspecto importante é a sua grande mobilidade (ChARles-Dominique et al. 1981) e ampla distribuição geográfica na América do Sul, ocorrendo em grande parte do território brasileiro, passando pela Colômbia até o Paraguai (EISENBERg \& REDFord 1999).

Alguns estudos têm sugerido que as características estruturais do ambiente parecem influenciar a distribuição e a abun- dância dessa espécie de marsupial, podendo este fenômeno promover a redução da sua população em ambientes modificados (AlHo 1981, Pagglia et al. 1995). Além do mais, é importante ressaltar que este marsupial é encontrado em florestas secundárias, matas de galeria e plantações (EMmons \& FEer 1997), bem como na zona litorânea, associado às florestas de manguezal (Andrade \& Fernandes 2005).

$\mathrm{Na}$ tentativa de melhor entender a associação de $M$. demerarae com as florestas costeiras, o presente estudo traz a primeira abordagem sobre o padrão de distribuição espacial, proporção sexual, sobrevivência, recrutamento e tamanho populacional, além de correlacionar esses parâmetros demográficos aos fatores abióticos e bióticos característicos das manchas de terra firme e, principalmente, das florestas de mangue.

Revista Brasileira de Zoologia 24 (2): 271-276, junho 2007 


\section{MATERIAL E MÉTODOS}

\section{Área de estudo}

A área de estudo, denominada "Fazenda das Salinas" $\left(046^{\circ} 40^{\prime} 20,4^{\prime \prime} \mathrm{W}\right.$ e $\left.0^{\circ} 55^{\prime} 26,7^{\prime \prime} \mathrm{S}\right)$, está situada nas planícies elevadas da península bragantina à aproximadamente $20 \mathrm{~km}$ da cidade de Bragança, Pará. Ao longo dos $166 \mathrm{~km}^{2}$ da península (LARA 2003) são encontrados diversos ambientes que incluem dunas, praias, campos salinos e bosques de terra firme, sendo que apenas estes dois últimos, juntamente com as florestas contíguas de mangue, compõem a paisagem vegetacional da área de estudo (Fig. 1).

Os manguezais bragantinos são dominados pelas espécies arbóreas Rhizophora mangle Linnaeus, Avicennia germinans (Linneus) Stearn. e Laguncularia racemosa (Linnaeus) Gaertn. f., ao passo que nas "ilhas" de terra firme, já foram catalogadas espécies das famílias Simaroubaceae, Myrtaceae, Arecaceae, Sapindaceae, Lecythidaceae, Arecaceae, Burseraceae, Ericaceae e Cecropiaceae (M.M.O. Abreu dados não publicados), sendo esta última um forte indicativo de mata secundária.

Os dados climáticos utilizados neste trabalho foram cedidos pelo Departamento de Meteorologia da Universidade Federal do Pará (UFPA) e são provenientes da Estação Meteorológica Automática, localizada no Furo Grande (0142'30"S e 051ํ31'51”W), cerca de $10 \mathrm{~km}$ da Fazenda das Salinas.

\section{Captura-marcação-recaptura}

Foram realizadas coletas bimestrais durante o período de novembro/2002 a setembro/2003. Cada campanha foi constituída por quatro noites de captura, com 160 armadilhas do tipo Tomahawk de 30x12x12 cm. Estas foram distribuídas em intervalos de $10 \mathrm{~m}$ ao longo de quatro transecções de $400 \mathrm{~m}$ cada (T1 e T2 na terra firme e T3 e T4 no manguezal - Fig. 1 e Tab. I). Nesta área foi instalado um total de 80 armadilhas para cada ambiente. $\mathrm{Na}$ terra firme, as armadilhas foram dispostas de forma alternada no chão da floresta e fixadas a $3 \mathrm{~m}$ de altura nas árvores, ao passo que no manguezal as armadilhas foram dispostas somente nas árvores, em função da mobilidade e constante inundação do chão da floresta pelas marés. Como iscas foram utilizados pedaços de abacaxi e/ou a mistura de pasta de amendoim, bacon e aveia.

As vistorias foram realizadas ao amanhecer (aproximadamente 05:30 h), sendo anotados o local e o número das armadilhas que continham espécimes capturados. Esses animais foram individualmente mantidos em sacolas de pano para obtenção de amostras fecais. Posteriormente, foram identificados com tatuagem numérica na base da cauda, bem como foram definidas: classe etária, peso, sexo, condição reprodutiva e medidas morfométricas (ex. comprimento do corpo, cabeça, cauda, pata posterior e orelha). Fêmeas e machos com menos de 62 g e 65 g, respectivamente, foram classificados como jovens ou subadultos (Meservé \& Le Boulegé 1987, Tribe 1990). A categoria Reprodutivo foi definida através da presença de fêmeas lactantes, grávidas ou com presença de filhotes no marsúpio, enquanto para os machos prevaleceu a ocorrência da descida testicular (EMmONs \& FeER 1997).

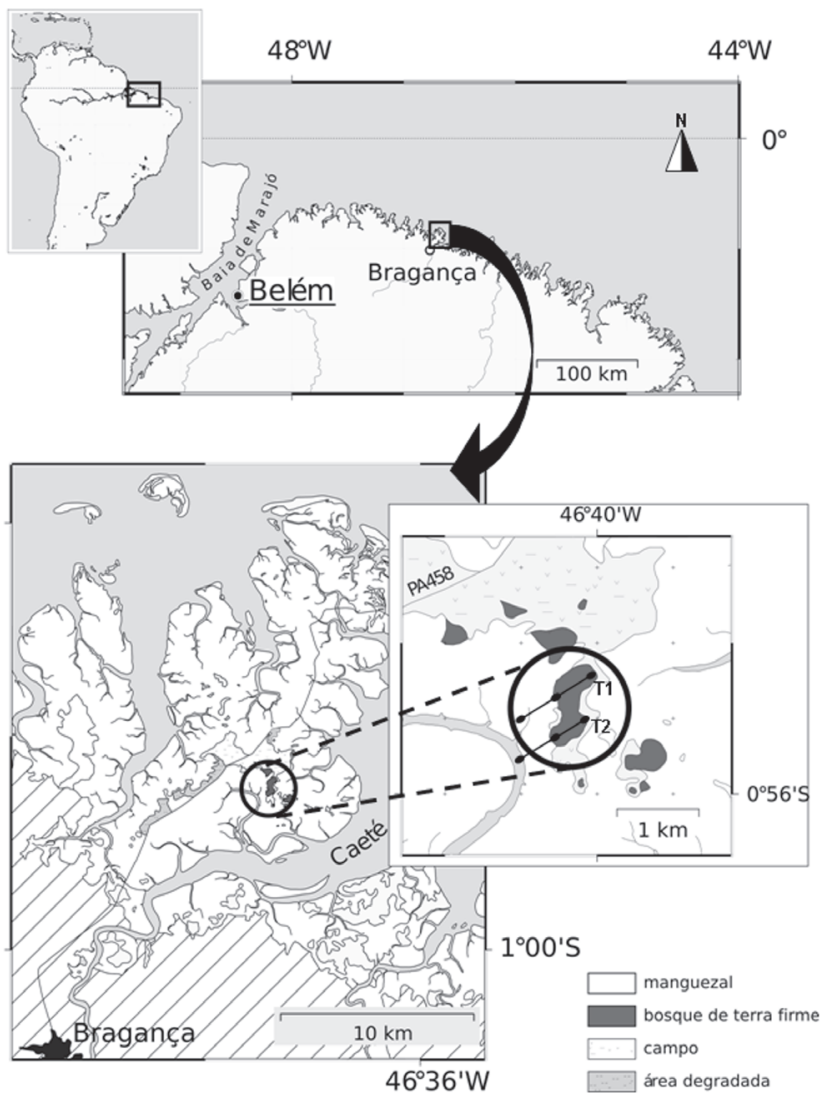

Figura 1. Localização da área de estudo na península bragantina, destacando as quatro transecções abertas na Terra Firme (T1 = Transecção 1 e T2 = Transecção 2) e no Manguezal (T3 = Transecção 3 e T4 = Transecção 4). Modificado de Mehlıc (2001).

Tabela I. Coordenadas geográficas das transecções de trabalho nos dois ecossistemas na Fazenda das Salinas, Bragança-Pará.

\begin{tabular}{cccc}
\hline Ecossistema & Transecção & Longitude (W) & Latitude (S) \\
\hline \multirow{2}{*}{ Terra-firme } & T1 & $46^{\circ} 40^{\prime} 11,5^{\prime \prime}$ & $0^{\circ} 55^{\prime} 21,3^{\prime \prime}$ \\
& T2 & $46^{\circ} 40^{\prime} 14,4^{\prime \prime}$ & $0^{\circ} 55^{\prime} 33,4^{\prime \prime}$ \\
\multirow{2}{*}{ Manguezal } & T3 & $46^{\circ} 40^{\prime} 20,4^{\prime \prime}$ & $0^{\circ} 55^{\prime} 26,7^{\prime \prime}$ \\
& T4 & $46^{\circ} 40^{\prime} 23,2^{\prime \prime}$ & $0^{\circ} 55^{\prime} 43,3^{\prime \prime}$ \\
\hline
\end{tabular}

\section{Análise dos dados}

O esforço de captura foi representado pelo total de armadilhas-noite. Para o cálculo do sucesso de captura em cada ambiente (Fazenda das Salinas/Manguezal/Terra Firme), o total de capturas foi multiplicado por 100 e o resultado dividido pelo valor do esforço amostral.

A razão sexual entre os animais também foi determinada, sendo em seguida utilizado o $\chi^{2}$ (com correção de Yates) para testar se os valores estimados em cada ambiente corres- 
pondiam ao esperado (50\% machos e 50\% fêmeas).

Como o estudo foi conduzido ao longo de um ciclo anual, uma análise de regressão linear serviu para avaliar a flutuação sazonal dos indivíduos capturados, através da relação entre o número total mensal de capturas e as taxas de precipitação.

As taxas de recrutamento, sobrevivência e tamanho da população foram estimadas através do método Jolly-Seber (JoLLy 1965, Caughley 1977, Krebs 1989). O teste de Lilliefors foi utilizado para testar a normalidade dos dados, sendo em seguida gerada uma matriz de correlação de Pearson (r), incluindo o tamanho populacional, recrutamento, sobrevivência e valores de precipitação local. A biomassa (g.ha) da população foi estimada mensalmente multiplicando-se a média da densidade (ind.ha ${ }^{-1}$ ) pela média do peso corpóreo (g) (Bergallo 1994).

As análises estatísticas foram realizadas com o uso dos programas estatísticos: Ecological Methodology v5.2 (Kenney \& Krebs 2000) e BioEstat 3.0 (Ayres et al. 2003).

\section{RESULTADOS}

Foram realizadas 153 capturas de 106 indivíduos de $M$. demerarae que resultou em um esforço amostral de 3.840 para a área da Fazenda das Salinas (FS), perfazendo um sucesso de captura de $4,0 \%$. Nos bosques de mangue $(\mathrm{M})$ o número de capturas foi 62 , proporcionando um sucesso de captura de $1,6 \%$. Já na terra firme (TF), com 91 capturas, o sucesso de captura foi de 2,4\%.

$\mathrm{Na}$ avaliação das categoriais sexuais foram registrados mais indivíduos machos do que fêmeas (Fig. 2), sendo essa diferença altamente significativa em quase todos os ambientes (FS: $1: 2 ; \chi^{2}=18,03 ; \mathrm{gl}=1 ; \mathrm{p}<0,001 / \mathrm{M}: 1: 3 ; \chi^{2}=24,39 ; \mathrm{gl}=1$, $\mathrm{p}<0,001)$, com exceção da terra firme, que muito embora tenha sido o ecossistema com maior número de indivíduos capturados (57\%), não apresentou diferença significativa entre machos e fêmeas de (TF: $1: 1 ; \chi^{2}=1,78 ; \mathrm{gl}=1$, $\left.\mathrm{p}>0,05\right)$. Dos indivíduos capturados, 42 foram considerados reprodutivos, dos quais $83 \%$ foram capturados durante a estação seca, principalmente na TF (69\%), sendo $43 \%$ de fêmeas e $57 \%$ de machos.

Os resultados também apontaram a ocorrência de variação sazonal na abundância dos indivíduos ao longo do ciclo anual (Figs 3 e 4). A análise de regressão mostrou que não houve diferença significativa entre o número de indivíduos e a precipitação $(r=0,69 ; n=6 ; p>0,05)$. As estimativas referentes ao tamanho populacional, à sobrevivência e ao recrutamento em cada ambiente mostraram que os valores das médias para esses parâmetros foram maiores na terra firme do que no manguezal (tamanho populacional, $\bar{x}=2,53$; sobrevivência, $\bar{x}=0,42$ e recrutamento, $\bar{x}=1,96$ ) (Tab. II e Fig. 5).

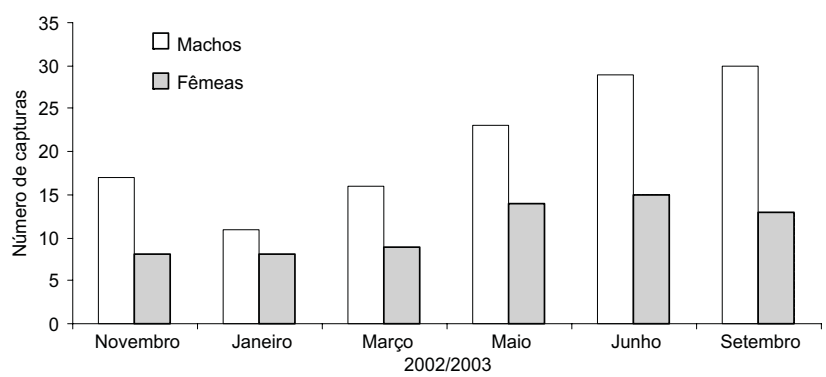

Figura 2. Número absoluto de machos e fêmeas da espécie $M$. demerarae, ao longo de todo o período de estudo (novembro/ 2002 a setembro de/2003), na Fazenda das Salinas, Bragança, Pará.
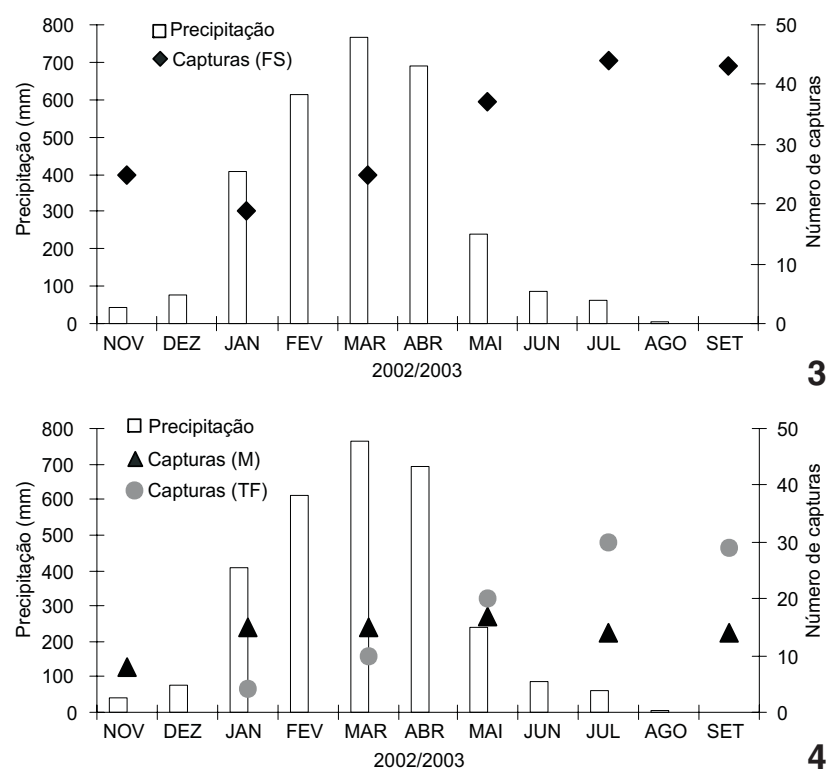

Figuras 3-4. Número de capturas de indivíduos da espécie $M$. demerarae e valores de precipitação por mês durante todo o período de estudo: (3) Fazenda das Salinas (FS); (4) os ecossistemas manguezal (M) e terra firme (TF).

Tabela II. Parâmetros populacionais de M. demerarae na Fazenda das Salinas e seus ambientes contíguos de manguezal e terra firme, Bragança, Pará. (DP) Desvio Padrão, (CV) coeficiente de variância.

\begin{tabular}{|c|c|c|c|c|c|c|c|c|c|}
\hline \multirow{2}{*}{ Ambiente } & \multicolumn{3}{|c|}{ Tamanho Populacional } & \multicolumn{3}{|c|}{ Sobrevivência } & \multicolumn{3}{|c|}{ Recrutamento } \\
\hline & Média & DP & CV (\%) & Média & DP & CV (\%) & Média & DP & CV (\%) \\
\hline Fazenda Salinas & 85,05 & 87,02 & 102,31 & 0,51 & 0,53 & 105,09 & 84,00 & 101,57 & 119,79 \\
\hline Manguezal & 1,90 & 1,53 & 80,83 & 0,22 & 0,27 & 120,30 & 1,23 & 1,39 & 112,70 \\
\hline Terra-firme & 2,53 & 2,22 & 87,69 & 0,42 & 0,43 & 103,76 & 1,96 & 2,25 & 114,45 \\
\hline
\end{tabular}



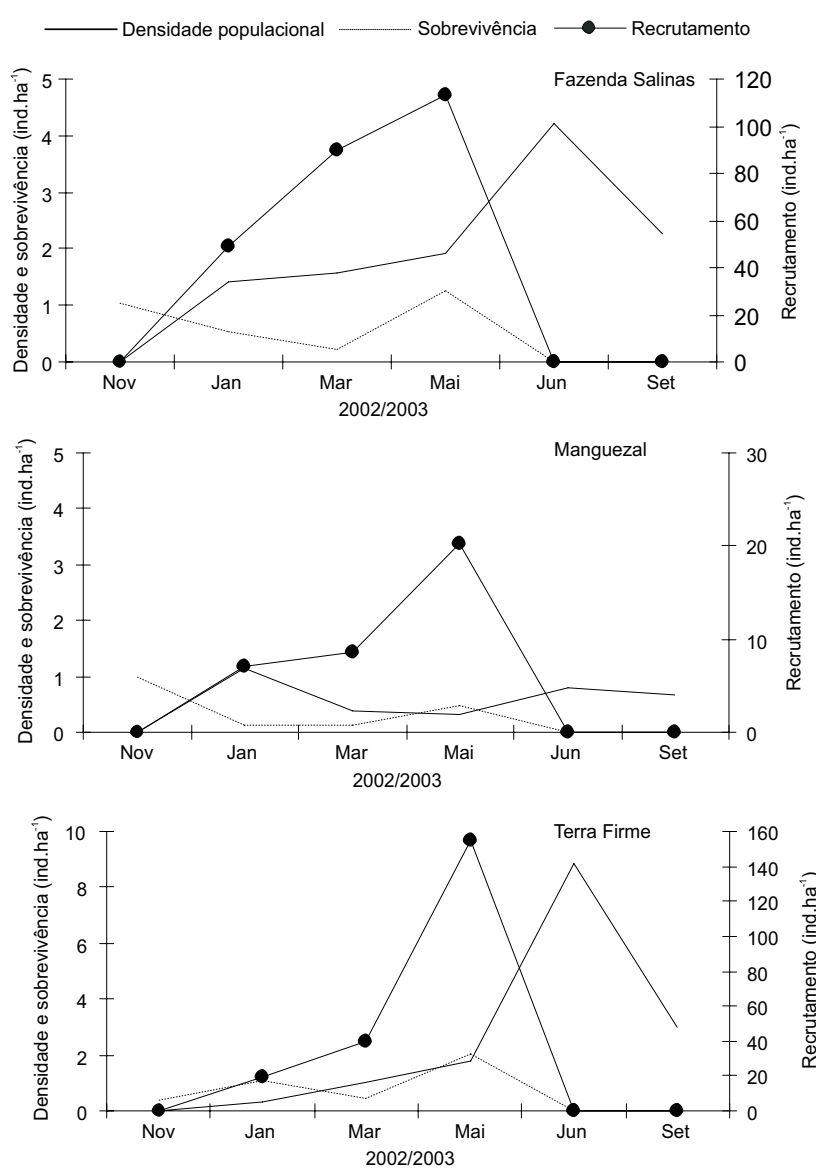

Figura 5. Parâmetros populacionais (densidade, sobrevivência e recrutamento) e suas variações para $M$. demerarae na Fazenda das Salinas, Bragança, Pará.

As análises de correlação entre os parâmetros demográficos (tamanho populacional, sobrevivência e recrutamento) e a precipitação não foram significativas para a FS e o ecossistema manguezal (Tab. III). Por outro lado, a análise entre os parâmetros sobrevivência e recrutamento apresentou correlação significativa $(\mathrm{r}=0,86 ; \mathrm{n}=6 ; \mathrm{p}<0,05)$ para o ecossistema TF (Tab. III).

\section{DISCUSSÃO}

Os valores do sucesso de captura, obtidos no presente trabalho, indicaram que o esforço empreendido ao longo do ciclo anual para amostrar as populações de $M$. demerarae foi semelhante aqueles apresentados em outros trabalhos com mamíferos terrestres (AdLer et al. 1997, Grelle 2003).

Para alguns autores como Shaeffer \& TAMmarin (1973), Krebs \& Myers (1974) e Lima et al. (2001), a demografia de muitas espécies de mamíferos seria marcadamente afetada por uma sazonalidade. Os dados do presente estudo tendem a corroborar esta hipótese, muito embora não tenham sido estatistica- mente significativos. Da mesma forma, os resultados mostraram não haver correlação entre precipitação e alguns fatores bióticos como: densidade (Andrade \& Fernandes 2005), disponibilidade de frutos e de insetos para M. demerarae, na Fazenda da Salinas (E. H. N. Chagas dados não publicados), corroborando com o presente estudo. De fato, o padrão de distribuição dos indivíduos em cada tipo de floresta, poderia ser gerenciado pelas características de cada ecossistema.

Quental et al. (2001) relataram que o padrão da dinâmica populacional seria reflexo do padrão reprodutivo, onde a sazonalidade deste último apresentaria variações, mais em função das taxas de recrutamento do que das taxas de sobrevivência, as quais são quase sempre constantes. Na Fazenda das Salinas, o maior número de capturas ocorreu durante a estação seca, embora os picos de recrutamento nos dois ambientes ( $M$ e TF) tenham sido registrados no final do período chuvoso, coincidindo com o período de menor disponibilidade de recurso. Assim, é importante ressaltar que os dados do presente estudo não corroboram a hipótese de que a disponibilidade de recursos é mais importante para a sobrevivência do que para o recrutamento, mesmo sendo os valores de sobrevivência correlacionados com as taxas de recrutamento.

Gentile et al. (2000) também relatam que a taxa de sobrevivência está relacionada a maior abundância de recursos, ocorrendo "o período crítico", neste caso, durante ou após a estação chuvosa. No caso da Fazenda das Salinas, a tendência sazonal das taxas de densidade da população de $M$. demerarae foi provavelmente influenciada pelas taxas de sobrevivência dos indivíduos, os quais permaneceram durante o período crítico, certamente nos bosques de terra firme, já que as taxas de recrutamento foram nulas para ambos os ambientes. Assim, é de se esperar que os dois ecossistemas (manguezal e terra firme) atuam como fontes diferenciadas de recursos para esses marsupiais.

Considerando os resultados apresentados no presente estudo, é razoável pensar que a terra firme atua como a principal fonte de recursos para esses marsupiais, principalmente pelo fato de que normalmente esse sistema apresenta maior diversidade e densidade de frutos e insetos do que o manguezal. Este último, por sua vez, parece atuar não apenas como um corredor de acesso às "ilhas" de terra firme, mas também como uma fonte secundária de recursos alimentares, principalmente de insetos.

Por fim, registros complementares (fotografias e avistamentos) de espécimes de $M$. demerarae em diferentes pontos dos manguezais na península bragantina, confirmam que esta espécie de marsupial utiliza diferentes áreas de manguezal muito distante das "ilhas" de terra firme onde o presente estudo foi realizado, corroborando a hipótese de Moraes Jr \& Chiarello (2005), na qual a extensão da área de vida destes animais deve ser muito maior do que se tem registro.

\section{AGRADECIMENTOS}

Ao Projeto Milênio e à Universidade Federal do Pará, pelo apoio financeiro. Ao Conselho de Desenvolvimento Científico 
Tabela III. Correlação de Pearson (r) entre os parâmetros populacionais de M. demerarae e os valores de precipitação, Bragança, Pará.

\begin{tabular}{|c|c|c|c|c|c|}
\hline Ambiente & Parâmetros populacionais & Tamanho Populacional & Sobrevivência & Recrutamento & Precipitação \\
\hline \multirow[t]{4}{*}{ Fazenda das Salinas } & Tamanho populacional & 1 & - & - & - \\
\hline & Sobrevivência & $-0,2720$ & 1 & - & - \\
\hline & Recrutamento & 0,1115 & 0,4480 & 1 & - \\
\hline & Precipitação & 0,0840 & $-0,0425$ & 0,7078 & 1 \\
\hline \multirow[t]{4}{*}{ Manguezal } & Tamanho populacional & 1 & - & - & - \\
\hline & Sobrevivência & $-0,4627$ & 1 & - & - \\
\hline & Recrutamento & 0,1088 & 0,0737 & 1 & - \\
\hline & Precipitação & 0,3196 & -2125 & 0,4486 & 1 \\
\hline \multirow[t]{4}{*}{ Terra-firme } & Tamanho populacional & 1 & - & - & - \\
\hline & Sobrevivência & 0,1949 & 1 & - & - \\
\hline & Recrutamento & 0,3893 & 0,8670 & 1 & - \\
\hline & Precipitação & 0,5595 & 0,6165 & 0,7442 & 1 \\
\hline
\end{tabular}

e Tecnológico (CNPq), pela concessão de Bolsas de Iniciação Científica aos acadêmicos Elaine E.N. Chagas e Geovanny B. Lima, os quais auxiliaram nas campanhas de campo e no trabalho laboratorial. Ao Dr. José de Sousa e Silva Júnior (Museu Paraense Emílio Goeldi) e Dr. Alfredo Languth (Universidade Federal da Paraíba) pela identificação do material coletado. Ao CNPq pela concessão da Bolsa de Mestrado a F.A.G. de Andrade (Processo 390007/2004-8).

\section{REFERÊNCIAS BIBLIOGRÁFICAS}

Adler, G.H.; J.J. Arboledo \& B.L. Travi. 1997. Population dynamics of Didelphis marsupialis in northern Colombia. Studies on Neotropical Fauna and Environment 32: 7-11.

AlHo, C.J.R. 1981. Small mammal population of Brazilian Cerrado: the dependence abundance on diversity habitat complexity. Revista Brasileira de Zoologia 41: 223-230.

Andrade, F.A.G. \& M.E.B. Fernandes. 2005. Mamíferos terrestres e voadores, p. 103-126. In: M.E.B. Fernandes (Ed.). Os manguezais da costa norte brasileira. São Luís, Fundação Rio Bacanga, vol. 2, 156p.

Ayres, M.; M. Ayres Junior \& A.S. Santos. 2003. Programa BioEstat 3.0: aplicações estatísticas nas áreas das Ciências Biológicas e Médicas. Belém, Sociedade Civil Mamirauá, 290p.

Bergallo, H.G. 1994. Ecology of a small mammal community in an Atlantic Forest area of Southeastern Brazil. Studies on Neotropical Fauna and Environment 29: 197-217.

Caughley, G. 1977. Analisys of vertebrate populations. London, A Wiley-Intercience Publisher, 234p.

Charles-Dominique, P.; M. Atramentowicz; M. Charles-Dominique; H. Gerard; C.M. HLadik \& M.F. Prévost. 1981. Les mammiferes frugivores arboricoles nocturnes d'une forêt guyanaises: inter-relations plantes-animaux. Revue d'Ecologie Terre et la Vie, Paris, 35: 341-435

EISENBERG, J.F. \& K.H. REDFORD. 1999. Mammals of the neotropics: the central neotropics. Ecuador, Peru, Bolivia, Brazil. Chicago, The University of Chicago Press, 609p.

EMMONs, L.H. \& F. FeER. 1997. Neotropical rainforest mammals: a field guide. Chicago, The University of Chicago Press, XVI+307p.

Gardner, A.L. 1993. Order Didelphimorphia, p. 15-23. In: D.E. WILSON \& D.M. REEDER (Eds). Mammal species of the world: a taxonomic and geographic reference. Washington, Smithsonian Institution Press, 1312p.

Gentile, R.; P.S. D’Andrea; R. Cerqueira \& L.S. Maroja. 2000. Population dynamics and reproduction of marsupials and rodents in a Brazilian rural area: a five year study. Studies on Neotropical Fauna and Environment 35: 1-9.

GrelLE, C.E.V. 2003. Forest structure and vertical stratification of small mammals in a secondary Atlantic Forest, southeastern Brazil. Studies on Neotropical Fauna and Environment 35: 1-9.

JolLy, G.M. 1965. Explicit estimates from capture-recapture data with both death and immigration - stochastic model. Biometrika 52: 225-247.

Kenney, A.J. \& C.J. Krebs. 2000. Programs for ecological methodology, version 5.2. Available in the World Wide Web at: http://www.zoology.ubc.ca/ krebs [Accessed in 11.XI.2006]

KreBs, C.J. 1989. Ecological methodology. New York, Harper Collins Publishers, 652p.

Krebs, C.J. \& J.H. Myers. 1974. Population cycles in small mammals. Advances in Ecological Research 8: 267-399.

LARA, R.J. 2003. Amazonian mangroves - a multidisciplinary case study in Pará State, North Brazil: Introduction. Wetlands ecology and management 11: 217-221.

Leite, Y.L.R.; J.R. Stallings \& L.P. Costa. 1994. Partição de recursos entre espécies simpátricas de marsupiais na reserva biológica de Poços das Antas, Rio de Janeiro. Revista Brasileira de Zoologia 54: 525-536. 
Lima, M.; R. Julliard; N.C. Stenseth \& F.M. Jaksic. 2001. Demographic dynamics of a neotropical small rodent (Phyllotis darwini): feedback structure, predation and climatc factors. Journal of Animal Ecology 70 (5): 761-775.

Meservé, P.L. \& E. Le Boulegé. 1987. Population dynamics and ecology of small mammals in the Northern Chilean semiarid region, p. 413-431. In: B.D. Patterson \& R.M. Timm (Eds). Studies in Neotropical mammalogy: essay in honor of Philip Hershkovitz. Chicago, Fieldiana Zoology, New Series, no. 39, 506p.

Moraes Jr, E.A. \& A.G. Chiarello. 2005. A radio tracking study of home range and movements of the marsupial Micoreus demerarae (Thomas) (Mammalia, Didelphidae) in the Atlantic forest of south-eastern Brazil. Revista Brasileira de Zoologia 22(1): 1-291.

Recebido em 29.III.2006; aceito em 24.IV.2007.
NoWAK, R.M. 1999. Walker's mammals of the world. Baltimore, The Johns Hopkins University Press, vol. 1, 1248p.

Pagglia, A.P.; P. de Marco Jr.; F.M. Costa; R.F. Pereira \& G. Lessa. 1995. Heterogeneidade estrutural e diversidade de pequenos mamíferos em um fragmento de mata secundária de Minas Gerais, Brasil. Revista Brasileira de Zoologia 12 (1): 67-79.

Quental, T.B.; F.A.S. Fernandez; A.T.C. Dias \& F.S. Rocha. 2001. Population dynamics of the marsupial Micoureus demerarae in small fragments of Atlantic Coastal Forest in Brazil. Journal of Tropical Ecology 17: 339-352.

Schaffer, W.M. \& R.H. TAmarin. 1973. Changing reproductive rates and population cycles in lemmings and voles. Evolution 27: 111-124.

Tribe, C.J. 1990. Dental age classes in Marmosa incana and other Didelphois. Journal of Mammalogy 71: 566-569. 